\title{
ORIGEN Y DESARROLLO DE LA TEORIA DEL CONOCIMIENTO
}

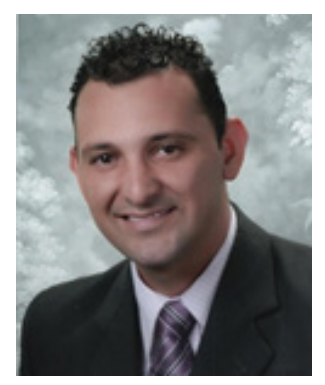

Julian Alberto Moncada Tarazona

Institución Educativa Estambul

Secretaria de Educación de Manizales (Caldas, Colombia)

jamta1990@hotmail.com

Fecha de recepción: 06/04/2019

Fecha de revisión: 05/05/2019

Fecha de aceptación: 23/05/2019

\section{RESUMEN}

Para empezar con el abordaje de esta temática, se hace necesario contextualizar al lector con el fin de guiarlo hacia un proceso de comprensión más dinámico, por tal razón, como primer momento se aterrizará el concepto de filosofía para consolidar unas bases teórico-cognitivas fuertes y a partir de allí se ira avanzando en complejidad hasta el punto de brindar algunas herramientas necesarias y pertinentes para realizar un proceso de exegesis frente a la Génesis y el desarrollo de la teoría del conocimiento. Posteriormente se analizará el origen del conocimiento iniciando un trasegar histórico desde los primeros seres humanos, pasando por la Grecia clásica y conociendo a los filósofos naturalistas, los filósofos presocráticos, y los 3 grandes filósofos griegos universales (Sócrates, platón y Aristóteles). Acto seguido, se conocerá el desarrollo de la filosofía en la edad media, dado por una fusión cultural que derivo en unas transformaciones sociales y filosóficas propias de las condiciones que se afrontaban y que por lo general giraban en torno a la concepción Teocentrista. Avanzando cronológicamente, se abordará la edad moderna o época de descubrimiento, donde surgen movimientos filosóficos que van en contra del periodo inmediatamente anterior y donde se privilegia una nueva tendencia hacia la razón. Por último, se aborda la época contemporánea como momento de auge científico que trae consigo una crisis social y una tensión global, producto de las consecuencias del desarrollo desenfrenado, que para algunos no representa un progreso para la humanidad.

Palabras clave: Teoría del Conocimiento, historia de la filosofía, Teocentrismo 


\title{
ORIGIN AND DEVELOPMENT OF THE THEORY OF KNOWLEDGE
}

\begin{abstract}
To start with the approach to this topic, it is necessary to contextualize the reader in order to guide it towards a more dynamic process of understanding, for this reason, as a first moment, the concept of philosophy will be landed to consolidate strong theoretical-cognitive bases from There it will progress in complexity to the point of providing some necessary and relevant tools to carry out an exegesis process about Genesis and the development of the theory of knowledge. Afterwards, the origin of knowledge will be analyzed starting with a historical journey from the origin of the first human beings, next, through classical Greece, and meeting the naturalist philosophers, the preSocratic philosophers, and the 3 great universal Greek philosophers (Socrates, Plato and Aristotle). Then, the development of philosophy in the Middle Ages will be known, given by a cultural fusion that led to social and philosophical transformations typical of the conditions that were faced and that generally revolved around the Theocentric conception. Moving forward chronologically, the modern age or epoch of discovery will be addressed, where philosophical movements that go against the immediately previous period arise and where a new trend towards reason is privileged. Finally, the contemporary era is approached as a moment of scientific boom that brings with it a social crisis and global tension, a product of the consequences of unbridled development, which for some does not represent progress for humanity.
\end{abstract}

Keywords: Theory of Knowledge, history of philosophy. Theocentrism

\section{INTRODUCCIÓN}

La naturaleza humana se caracteriza por esa necesidad de conocer el mundo que lo rodea, esa exigencia por comprender e intervenir las realidades con el fin de generar una mayor adaptabilidad y facilitar el modo de habitar y existir ese mundo. Es así, como desde sus inicios el hombre de forma perentoria ha estudiado los diferentes fenómenos con los que cohabita el mundo, hasta el punto de representar y dar sentido a la naturaleza a través del conocimiento. Este proceso de entendimiento ha sido influenciado por diferentes paradigmas filosóficos que han impactado los procesos históricos $\mathrm{y}$, por ende, son elementos que fundamentaron la construcción de sociedad que hoy conocemos.

Así entonces, se hace importante explicar el término de filosofía para empezar a delimitar el fin de este ensayo, con este fin, es importante volver a las raíces etimológicas de la palabra para comprender su potencial semántico. En este sentido, Corominas (1987) en su diccionario 
etimológico plantea que el termino se deriva de la palabra griega philéo que significa "yo amo" y de la palabra griega sophia que significa "sabiduría o ciencia" (p.604). Como podemos ver, al hacer un análisis podemos apreciar como el concepto se direcciona a ese afecto y cariño que se genera hacia el conocimiento, dicho de otra manera, es la reflexión sobre la naturaleza del mundo tangible e intangible, su origen, sus atributos, sus fenómenos y especialmente la humanidad con respecto a su existencia y su forma de vivir la particularidad dentro de la universalidad, con la única pretensión de encontrar, demostrar o significar algo.

Con respecto a esta definición, Piaget citado por Quintana (1982) expone, "la filosofía consiste en una búsqueda de lo absoluto o en un análisis de la localidad de la experiencia humana" (p.66), así mismo Quintana (1982) aclara que, "El filósofo la hace de una manera sistemática y profunda, pero ningún hombre deja de hacerla, aunque sea a un nivel superficial y poco consciente: al ser el hombre racional, siempre elabora una razón de ser de las cosas y una justificación de sus actos, con lo cual no puede evitar el filosofar" (p.66) Así entonces, este recorrido al tratarse de un proceso de estudio de la realidad se constituye en una forma de modelarla u ordenarla a través del entendimiento y de la construcción de un nuevo conocimiento.

\section{DESARROLLO}

\section{Génesis de la filosofía en la edad antigua}

La historia de la humanidad ha demostrado que el sendero transitado por el ser humano en busca de la comprensión es arduo y extenso, la búsqueda del conocimiento es un aspecto que ha generado un gran interés en la aventura de la humanidad. Si retrocedemos en el tiempo encontraremos que somos descendientes de los primeros homos sapiens o hombres pensantes, quienes fueron los primeros homínidos con la capacidad de generar imágenes mentales y conceptos de la realidad, con esta nueva condición dada por la evolución el hombre emprendió el camino hacia la comprensión del mundo.

El cumulo de ideas y representaciones que fueron construyendo los antepasados humanos, trajo consigo la edificación de la cultura y dio inicio a una nueva forma de ver y habitar el mundo, característica que le permitió al hombre adaptarse con más facilidad al medio y establecerse en nuevos lugares donde con anterioridad se consideraba imposible vivir, esto le brindo, la posibilidad de expandirse y prosperar en todos los rincones del planeta.

Los pensamientos primitivos de los primeros seres humanos donde solo intentaban comprender el mundo para poder sobrevivir a las tensiones que este les ofrecía son transformados 
por un nuevo estilo de vida más tranquilo y predecible producto del conocimiento acumulado que permite entender cómo funciona la tierra, esto desemboca en que algunos hombres desarrollen una nueva faceta intelectual en la cual pueden dedicarse a pensar fuera de la necesidad de satisfacer sus necesidades básicas o primarias. Este primer momento, que surge dentro de la incertidumbre y que siente ese afán por entenderlo todo, trae consigo una corriente de pensamiento llena de mitología, elemento que brindaba seguridad frente a lo desconocido.

Posteriormente, las primeras civilizaciones fueron un centro de desarrollo de conocimiento, pero en especial fue en Grecia donde se dio el origen a la filosofía y al conocimiento estructurado, esta perspectiva es compartida por Chacón (2017) quien plantea que "Los griegos, a partir de los filósofos de la Naturaleza (Tales, Anaximandro y Anaxímenes), marcaron la pauta de la Filosofía al independizarla de la religión e iniciar, así, una nueva forma de conocimiento en la que el objeto del pensamiento apareció como aquello que realmente es, es decir, en sus cualidades esenciales". (p.242)

En este sentido, la Filosofía surge de la condición y disposición del hombre para construir conceptos frente a la realidad que le permitieron tomar ventaja de las posibles situaciones que afrontaba, en este sentido el origen filosófico está en las primeras observaciones naturales que lo llevaron meditar sobre el mundo tangible o natural. Es así, como a partir de Tales de Mileto a quien ya podemos ubicar temporalmente en el siglo VII a.c. se transforma la forma de percibir el mundo y se proyecta una nueva línea de pensamiento racional a través de la cual se busca una explicación dada desde la razón y no desde el mito.

Un claro ejemplo de su aporte al conocimiento, es que propuso que el elemento original y origen de todas las cosas que existen es el agua, cambiando el orden en cómo se percibían las cosas al desmitificar la creación, además de generar una corriente de pensamiento que podría decirse se mantiene hasta el día de hoy y es que, dentro de la diversidad, la diferencia, la multiplicidad siempre hay un principio u origen único de todo, como lo dice Perea citado por Chacón (2017) “... de esta manera, en Tales de Mileto la filosofía da por vez primera una interpretación unitaria y al mismo tiempo dinámica del ser: sin dejar de reconocer la multiplicidad y variedad de las cosas, las reduce a cierta unidad, mediante el concepto de 'principio'." (p.242).

En una línea de pensamiento similar y me refiero a la concepción naturalista que busca el origen de todas las cosas en la naturaleza, están Anaximandro quien vivió entre el siglo VI y V a.c. y Anaxímenes quien vivió en el siglo $\mathrm{V}$ a.c., el primero discípulo de Tales quien en su observación metódica define que el origen de todo es el Arje una partícula única e inalterable, además, a través 
de su reflexión se acerca a muchos conceptos demostrados científicamente en la actualidad. Por otro lado, Anaxímenes, discípulo de los dos anteriores propone el Aire como elemento original quien apoya su teoría en lo que hoy podemos denominar cambios de estado de la materia.

Continuando de forma cronológica, aparece Pitágoras quien vivió entre el siglo VI y V a.c. De igual forma que los filósofos anteriores, busca entender el principio y origen del mundo y el universo, pero en este caso abandona el plano natural y físico de los anteriores,

Según Chacón (2017) "El pensamiento de Pitágoras se dividió en dos aspectos que son: el mítico y el científico; en el aspecto mítico creyó en la inmortalidad y en la transmigración del alma, pensamiento que fue recuperado, posteriormente, por Platón y, en el aspecto científico, se interesó tanto por la forma o estructura del Mundo, como por su principio material. Considerando, así, que la esencia permanente del Universo se encuentra en los principios matemáticos, fundamentalmente en los números. Esto significa que, para Pitágoras, el número es la esencia de todas las cosas, porque el número ocupa un lugar intermedio entre la percepción sensible y las ideas”. (p.243)

Igualmente, en el siglo VI a.c. destaca el filósofo Parménides de Elea, para Chacón (2017) este,

"le dio un movimiento dialéctico al pensamiento, es decir, a diferencia de los anteriores, se desprendió de la forma sensible y de la forma del número y planteó, por primera vez, la relación entre la realidad y la razón. Esto quiere decir que el pensamiento se convirtió en libre y para sí; Parménides empezó a hablar específicamente del conocimiento y, al respecto, señala que el único camino para llegar a éste es la razón y el Ser, considerando a este último como inmutable, eterno, indivisible, homogéneo e inmóvil y que, además, no tiene principio ni fin, es decir, para este pensador, el Ser no puede originarse del no Ser, además, éste ni surge ni desaparece”. (p.243)

En este mismo periodo, Heráclito propone que el mundo y el universo están gobernados por la ley de la razón, y para comprender ese mundo es necesario hacer uso de los sentidos, pero de igual manera es necesario comprender lo que se percibe con la inteligencia y con el alma. "Puede señalarse que fue uno de los primeros filósofos que le dio especial importancia a la conciencia, al señalar que el entendimiento no es otra cosa que la interpretación o, dicho de otra manera, el modo de la ordenación del todo. Por esta razón, en la media en la que un sujeto participa de su conocimiento, está en el camino de la búsqueda de la verdad; en cambio cuando sólo se sabe lo propio y peculiar, se está en el error” (Chacón, 2017, p.244) 
En el siglo $\mathrm{V}$ antes de cristo encontramos a Sócrates quien es considerado como una de las figuras más importantes entorno a la filosofía, fue tanta su influencia, que se consolido como un referente histórico y en algunos textos se encuentra el termino de presocráticos para referirse a los pensadores anteriormente mencionados. Su gran aporte fue darle el valor de ciencia a la filosofía, y era esta la encargada de ver la esencia del ser humano y no al contrario, por otro lado, edifico un método denominado mayéutica, en el maestro lleva al alumno a que descubra por sí mismo las verdades a través de preguntas apropiadas que lo direccionaran en el camino del entendimiento.

Frente al anterior punto, Hegel citado por Chacón (2017) dispone:

"Puede decirse que, con Sócrates, aparece el saber; con él ya no existe la preocupación de saber que es la Naturaleza, sino qué es la verdad. La esencia ahora ...se determina, no como el ser en sí, sino como lo que es en el conocimiento. Vemos, pues, cómo aparece el problema de la relación entre el pensamiento consciente de sí mismo y la esencia y cómo este problema se convierte en el más importante de todos" (p.246).

Siguiendo con este análisis cronológico, encontramos a Platón quien vivió en el siglo IV a.c. Fue discípulo de Sócrates y es también considerado como uno de los grandes filósofos universales. Su influencia en la filosofía está relacionada con el concepto de que los sentidos no ofrecen una imagen o idea real, completa o verídica del mundo, además agrega que la única forma de comprender la realidad es a través del uso de la razón y el entendimiento.

"Otra cosa para resaltar es que hasta Platón cuerpo y alma eran uno solo, por lo que el ejercicio de la sabiduría partía de esta premisa. Con Platón el alma adquiere un mayor estatus que el cuerpo, pero ninguno de los dos puede ser considerado como despreciable, por eso Platón recomienda el ejercicio físico (cuerpo sano, mente sana), la moderación, el amor propio, entre otros como garantía de la salud". (Botero, 2001, p.2)

Así mismo Chacón (2017) sostiene que:

"Platón estuvo convencido que el conocimiento puede alcanzarse, ya que el conocimiento debe ser certero e infalible, además, debe tener como objeto lo que es en verdad real, porque lo que es real tiene que ser fijo, permanente e inmutable, no obstante, lo real lo consideraba como lo ideal; lo cual se opone al mundo físico, por tanto, rechazó el pensamiento de los filósofos que afirman que todo conocimiento se deriva de la experiencia"(p.247).

En el siglo IV a.c. También se cuenta con otro de los grandes filósofos universales, Aristóteles 
discípulo de Platón, defiende la unión entre conocimientos y propone la filosofía como elemento articulador, por lo tanto, el científico debe explicar de manera universal y no particular los fenómenos. Así mismo plantea que la condición pensante del ser humano le hace sentir curiosidad frente a la naturaleza, la cual es explorada a través de los sentidos ofreciendo sensaciones que se van acumulando en la memoria y a la vez se consolidan en experiencias.

"Aristóteles afirma que el hombre conoce por medio de los sentidos, es decir, todo conocimiento comienza en los sentidos; mediante ellos, el hombre, toma contacto con las cosas, no obstante, el conocimiento no se agota en la experiencia; además, pudo distinguir varios niveles o grados de conocimiento que son: el conocimiento sensible, derivado directamente de la sensación, que es un tipo de conocimiento inmediato y fugaz, desapareciendo con la sensación que lo ha generado. El conocimiento sensible es propio de los animales inferiores. En los animales superiores, este conocimiento sensible, al relacionarse con la memoria sensitiva y con la imaginación, da lugar a un tipo de conocimiento más persistente. Este proceso de conocimiento es el que tiene lugar en el hombre, en el cual se genera la experiencia como resultado de la actividad de la memoria, no obstante, aunque este conocimiento no le permite conocer el por qué y las causas de los objetos conocidos, sí les permite saber que existen, es decir, la experiencia consiste en el conocimiento de las cosas particulares”. (Chacón, 2017. P.248)

\section{Desarrollo histórico filosófico: Edad Media}

El periodo de transición de la edad antigua a la edad media fue un proceso de cambio y transformación en todos los ámbitos sociales, en primer lugar, la expansión del imperio Romano trajo consigo la desaparición de varias culturas que caen ante la cultura hegemónica impuesta por el conquistador, tan solo algunas culturas logran mantenerse hasta el punto de generar una fusión con la cultura dominante como es el caso de la cultura griega, de ahí que las teorías filosóficas griegas sean parte fundamental de la filosofía universal.

Aun así, este proceso de fusión cultural y filosófica no fue positivo en lo relacionado al conocimiento, la expansión romana que inicia en el siglo VI a.c y alcanza su apogeo en el siglo II d.c. Fue seguida por la caída del imperio romano de occidente en el siglo V. Toda esta dinámica trajo consigo una perdida invaluable de los avances científicos, culturales y filosóficos de la edad antigua, ejemplo de esto es la evolución que tuvo la filosofía y el conocimiento en la antigua Grecia la cual se puede decir fue masacrada en el incendio de la biblioteca de Alejandría. Se puede 
afirmar, que de no haberse presentado esta perdida masiva de conocimiento tal vez la historia de la humanidad hubiera tomado otro rumbo.

Igualmente, los cambios de la época junto con la caída del imperio romano de occidente, abre las puertas a una nueva época denominada la edad media que supone transformaciones económicas, políticas y sociales. Con respecto a la filosofía, el conocimiento y la cultura se plantea que el periodo Greco-Romano llega a su fin, y de nuevo se somete a un paradigma teocéntrico por lo general cristiano que relega los avances sustanciales propuestos por los filósofos griegos.

En este sentido, “en el medioevo, según la tradición spengleriana, se pueden distinguir la temprana edad media (siglos III al IX), alta edad media (siglos X al XIII) y baja edad media (siglos XIV al XV)". (Botero, 2001, p.2). Otros autores como Lázaro (2018) proponen, una división temporal en dos épocas con la siguiente extensión, alta edad media (siglo VI a XI) y baja edad media (siglo XII al XIV). (p.1)

Con respecto al primer autor, "La temprana edad media se caracterizó por la cimentación del poder papal y el desprestigio de toda sabiduría con excepción de aquella destinada al conocimiento de Dios y su relación con los mortales (se desliga la teología de la sabiduría e incluso la hija se apodera de la madre, la teología arropa a la sabiduría misma)" (Botero, 2001, p.2).

Con respecto al primer momento llamado temprana edad media, se caracteriza como ya lo hemos dicho por ser el periodo de la caída del imperio romano que trajo consigo una serie de invasiones al imperio entre los siglos III al IX. En el siglo V d.c. los germanos invaden el imperio y se asientan en estos territorios y en el siglo VII la expansión musulmana avanza hacia el oriente y norte de África y a su vez logra conquistar las penínsulas ibérica y arábiga, la fuerte división política dentro del imperio y la perdida de territorios geográficos lleva a que se generen una serie de reformas en la que los pueblos se unifican frente al cristianismo como dogma y una religión oficial, con el fin de hacerle frente a estos invasores que son considerados como barbaros.

Esta situación causa una hibridación de las culturas quienes adoptan de nuevo la cultura hegemónica que se identifica por un valor exagerado de la teología sobre la filosofía. Como bien lo dice Botero (2001), "En el medioevo podemos afirmar la existencia de una filosofía cristiana en oposición a la concepción ilustrada y moderna que afirmaba que el medioevo fue un letargo de la humanidad" (p.2).

Aun en tiempos de crisis, algunos filósofos destacan por su labor en la búsqueda del 
entendimiento, este es el caso de San Agustín de Hipona quien vivió en el siglo V y fue considerado uno de los grandes pensadores del cristianismo quien adopta pensamientos de los filósofos griegos y los aplica al cristianismo, uniendo los temas de razón y religión. Así lo muestra Saeteros (2007) quien expone que "Dos son los únicos grandes temas que interesan a san Agustín: Dios y el alma. Como él mismo manifiesta, su más ardiente deseo va en esta dirección: que me conozca a mí y te conozca a ti” (p.190).

Seguidamente en la alta edad media, se continua la línea filosófica que deja la época anterior que se ocupa en pensar solo lo relacionado con Dios, y llega al punto de llamar profano todo conocimiento o búsqueda de este que no esté relacionado con Dios. Esta gran confianza en el aspecto mitológico y divino, produce un alejamiento de la razón, y produce una fragmentación disciplinar al no tenerse un objetivo filosófico claro, tan solo existía el componente teocéntrico como eje articulador que en su escasez de razón planteaba que todo era una gracia de Dios y no un proceso que requiera un análisis conceptual profundo. En concordancia Botero (2001) sostiene:

“El medioevo nace, pues, con desconfianza a la sabiduría como totalidad, lo cual afecta a la filosofía y la universidad naciente (que tuvieron que refugiarse en el estudio de la divinidad). Se inicia con la decadencia de la sabiduría la división entre las disciplinas, división que empieza a organizarse (y a reflejarse en las universidades) a partir de los objetos, es decir, se le asigna a la medicina el cuerpo, a la teología el alma, a la biología los animales no racionales, a la botánica las plantas, a las matemáticas la lógica y los números, a la astronomía el mundo supralunar (lo que está más allá de la luna que para todo medieval es el lugar donde encontramos la perfección de la creación divina), etcétera. Claro está que todas las áreas de estudio están subordinadas a la teología, allí empieza y muere todo intento de conocer, incluso la pretensión de universalidad que da origen a la universidad" (p.4).

Posteriormente sigue el periodo de la baja edad media, donde la mayoría de la población aún tiene la influencia de un pensamiento teocentrista, pero este pensamiento adoptaba ahora lo que Botero (2001) denomino "determinismo fatalista” (p.4) y empezó a atribuir las consecuencias de una realidad a la fortuna, no a un Dios, no a una ciencia, situación que puede describirse como un azar poco probable que no tiene explicación, hecho que se distancia de un proceso racional o intelectual. Citando a Botero (2001) encontramos que "La Fortuna, perdonen ustedes por hacer este énfasis, resume buena parte de la actividad de los siglos XIII y XIV, pues ya era común entre la población europea achacar sus bienaventuranzas y males a ella. Era ella quien definía la suerte de un individuo y de un pueblo" (p.4). 
Aun así, durante esta época surge una esperanza debido al gran progreso que se presenta en muchas áreas del saber, el interés por mejorar la calidad de vida y por entender la realidad, rescata de nuevo esa faceta intelectual que fortalece el desarrollo filosófico y científico, tarea que es efectuada por tan solo unas pocas personas. Así lo afirma Tamayo (2007), planteando que "Los estudios históricos sobre la Baja Edad Media permiten conocer la importancia de un incipiente "renacimiento" entre los siglos X y XIII, cuando hubo una renovación del pensamiento y la sensibilidad, y se estableció la reflexión basada en la argumentación y la razón lógica” (p.198).

Por otro lado, En esta época nos encontramos con grandes pensadores entre estos esta santo Tomas de Aquino, quien vivió en el siglo XIII, para este era importante entender que la razón no era suficiente para comprender a Dios, pero si era un elemento indispensable para comprender el mundo. Así también, es visto por Botero (2001), quien argumenta que,

"se recuperan las enseñanzas de Aristóteles por la autoridad de Santo Tomás de Aquino (denominado por sus enemigos como el obeso, quien fue canonizado por Juan XXII, el papa que permanece en Avignon como su sede apostólica y no en Roma como era la tradición) siendo tomadas sus enseñanzas como dogmas de fe en las universidades. Pues bien, Aristóteles propuso para su Atenas un modelo de ciencia de la causa final (no material) que se basaba en la demostración y no la experimentación, es decir, comprobar en la naturaleza que los juicios derivados de los silogismos eran ciertos y no experimentar para explicar las causas materiales y las leyes que rigen el objeto investigado (pretensión de la ciencia moderna)”. (p.5)

Esta época cierra con la caída del imperio Bizantino y con la invención de la imprenta, dos hechos que marcaron un hito en la humanidad el primero mostraba la edad media que había quedar atrás y el segundo representaba el inicio del progreso científico y filosófico al alcance de todos. Estos dos hechos históricos dieron lugar a un movimiento cultural denominado renacimiento en el cual se dejaba atrás el pensamiento del teocentrismo para que pasara a tomar protagonismo el antropocentrismo el cual desencadeno nuevas formas de ver el universo, el mundo y el hombre.

\section{La modernidad una avalancha de descubrimientos y desarrollo del pensamiento científico.}

La edad moderna que va desde el siglo XV hasta el siglo XVIII se destaca por ser la contraparte de la época vivida con anterioridad, La razón y el descubrimiento vuelven a cobrar importancia, y aunque no desaparecen las tradiciones religiosas, estas se separan de la racionalidad científica, además, se Conserva la fragmentación disciplinar donde cada una de las áreas se desarrolla de 
forma autónoma y por lo general sin construir correlaciones.

"La modernidad se caracterizó, en un principio, por una nueva mirada del conocimiento y por su cultivo. Cada uno de los demás conocimientos, como la medicina, el derecho, la biología, la física, la astronomía, entre otros, empiezan a florecer en las universidades independientemente bajo el auspicio del método de la experimentación y la explicación de las causas eficientes y materiales (no las finales, como era propio en la ciencia aristotélica), método que aportaron los físicos de la época, en especial F. Bacon y Newton. Así, van adquiriendo estatus científico y autonomía los saberes (en facultades dentro de la universidad) que antes se integraban en un todo”. (Botero, 2001, p.5)

Igualmente, La libertad humana se convierte en uno de los pilares del modernismo, a través de esta el hombre considera que puede ser artífice de su destino, el antropocentrismo dispone que el hombre es el actor principal de la obra llamada realidad, aun así, Botero (2001) afirma que "El hombre poco a poco pasa a ser servidor y no a ser servido. Las instituciones, tales como las científicas y las políticas, se entronan sobre el ser humano. "La modernidad libera al hombre del trasmundo y lo vuelve esclavo del intramundo. El hombre deja de ser criatura y se convierte en súbdito, pasa del dominio de la iglesia al dominio del estado" (p.7).

Se recupera el valor social de la investigación científica, devolviendo un estatus social a quienes se dedican a estudiar el mundo. Según Botero (2001), Con la adquisición del estatus científico por parte de múltiples áreas (o urdimbres) del saber, el sueño del sabio se ve reemplazado fácilmente por la realidad del hombre de ciencia o científico (se consolida un nuevo modelo antropológico: científico)" (p.6). Esto también lleva a que las disciplinas sean manejadas solo por expertos especializados que llevan a que el conocimiento en esas áreas se desarrolle más rápido, lo que genera una expectativa alta frente al desarrollo científico y tecnológico y sus implicaciones en el mejoramiento de la calidad de vida humana.

Durante esta época aparecieron científicos que evidenciaron el gran avance que trajo el fenómeno de la modernidad, en este sentido, se evidenciaron corrientes de pensamiento que llevaban a la adquisición del conocimiento. La primera de ellas es el renacimiento, descrita por Ramírez (2009) como:

"La concepción monacal del pensamiento no va acorde con el proceso de cambio y liberación de espíritus y mentes que ocurre en los ss XV y XVI, cambio que da lugar a la corriente transformadora llamada renacimiento, que como indica su nombre es un 're-nacer', es decir, volver-a-nacer y un volver la mirada a esa forma de conocer, 
entonces casi olvidada, que viene del mundo antiguo, especialmente de los griegos. Ese re-nacer se manifiesta en todas las formas del quehacer humano, artes, letras, ciencias, medicina y por supuesto en la búsqueda del conocimiento". (p.221)

Los principales pensadores de esta corriente moderna apostaban a los pensamientos racionales o sus opositores a los pensamientos empíricos. Por un lado, Descartes y Leibniz privilegiaban el racionalismo en la formalización de la ciencia, por lo que podríamos decir que su pensamiento sentía especial fascinación por la intuición, los números y el objetivismo. Por el otro, Locke y Hume preferían el empirismo y se inclinaban por el subjetivismo y la experiencia como fuente de conocimiento.

Otra corriente, propia de esta época es el positivismo de Comte, en la cual se "ensayan diseños de modelos matemáticos para conocer a priori posibilidades de ocurrencia de un determinado evento y crean pruebas de cálculo de probabilidades que aún hoy son paradigmas para el manejo de la estadística en investigación" (Ramírez, 2009, p.221), así mismo, "otra característica de la epistemología comteana es el monismo metodológico, entendido como la aplicación de un único método para analizar la ciencia, sea esta empírica o social" (Ramírez, 2009, p.222). esta corriente de pensamiento como ya se dijo, es parte esencial del método científico, donde se plantea que la única forma de acercarse al conocimiento es a través de la comprobación científica que se hace verificable por medio de un método.

Otro autor importante en la teoría del conocimiento moderno es Immanuel Kant quien transforma la perspectiva racionalistas y empiristas al cambiar el interés del conocimiento científico del objeto y asignárselo al sujeto.

"La epistemología kantiana conceptúa la subjetividad de los conceptos básicos de espacio-tiempo y la imposibilidad de llegar a representaciones puramente objetivas de cosas en sí mismas; para él, aun las categorías tomadas a priori (deducibles) son estáticas e independientes de la experiencia, de manera tal que solo tienen condición de posibilidad". (Ramírez, 2009, p.222)

\section{La contemporaneidad una época de desarrollo intelectual y científico.}

El desarrollo intelectual dado en la época moderna, desencadeno en el siglo XVIII un movimiento político, social y económico que cuestionaba la forma de gobierno de la monarquía absoluta, esto obviamente ligado a las condiciones de desigualdad a la que se enfrentaban las 
personas de la época, junto con una necesidad de libertades y derechos que garantizaran una calidad de vida digna para todas las personas por igual.

Este movimiento, denominado revolución francesa, transformo por completo en tan solo una década las dinámicas sociales del mundo. Las formas de gobierno se transformaron y dieron luz a nuevos estados y por ende se transformaron los territorios, la justicia, la política, la economía, la educación, la ciencia y muchos otros aspectos de carácter social. Ahora bien, aunque este movimiento trajo consigo la estructura social que hoy se vive, dando pie al régimen de derechos de todos los seres humanos, instaurando fundamentos de vida frente a la igualdad, libertad y fraternidad, situación que trae como consecuencia nuevos modelos de formación que están al alcance de toda la humanidad y que trae consigo un auge del conocimiento científico.

Frente a esta situación Botero (2001) plantea:

"Es por todos sabido que la cientificidad y su estado de exaltación general fundada en la prometida prosperidad acabó en dos cruentas guerras mundiales, imperialismos y colonialismos, maltrato a las naciones y pueblos débiles cobijados por el silencio de la historia, la destrucción sistemática del individuo y la explotación excesiva de los recursos naturales". (p.7)

Vemos entonces, como la perspectiva del desarrollo científico paso de ser la esperanza para mejorar la vida y se convierte en algunos casos en sinónimo de miedo e incertidumbre, estas nuevas dinámicas sociales que trae la contemporaneidad son motivo de análisis por los pensadores de la época, por este motivo aparecen corrientes de pensamiento que abordan las distintas situaciones emergentes en esta realidad.

Una de estas corrientes filosóficas emergentes en el en el siglo XIX es el materialismo dialectico de los autores Hegel, Marx y Engels, donde se plantea una estrecha relación entre el conocimiento y el mundo material de la época, en efecto la materia es considerada como la base absoluta del mundo y es la conciencia un elemento de esta que a su vez es un proceso organizado que posibilita su comprensión. "Para el materialismo, conciencia, espíritu y conocimiento solamente son posibles en cuanto el mundo es materia. El materialismo sostiene a la materia como sustrato de toda realidad, tanto objetiva -física- como subjetiva -pensamiento, conocimiento- y su concepción privilegia e independiza lo material de lo subjetivo" (Ramírez, 2009, p.222).

Otra corriente naciente es el existencialismo con autores como Heidegger, Sartre y Camus. Esta emerge como posibilidad de instituir una forma diferente de ver la realidad, planteando unos 
principios de libertad y responsabilidad individual que propone a la persona como edificador de su propia vida, por lo tanto, es solo en su particularidad que se le asigna significado al mundo, y es solo con la experiencia dada por la propia existencia que se puede construir el conocimiento. Así mismo, Botero (2001) plantea que "El existencialismo pone en duda la excesiva racionalidad del cientificismo, critica la mirada reduccionista del hombre que hace la ciencia, solicita de nuevo la intervención de la filosofía en los quehaceres de la cultura, afirma que no todo descubrimiento científico está destinado al "progreso humano" (Botero, 2001, p.7)

En otra línea de pensamiento aparece el pragmatismo con autores como James y Dewey. corriente que plantea una articulación entre teoría y práctica, en el cual el conocimiento se recopila de la práctica y se aplica a los nuevos procesos prácticos generando un ciclo de retroalimentación. Por otro lado, "propone crear modelos capaces de capturar 'toda' la información relevante y con esta data solucionar o descartar una hipótesis” (Ramírez, 2009, p.222).

El relativismo es otra tendencia filosófica de esta época, con autores como Habermars y Kraus. "El relativismo, una de las corrientes radicales del post-modernismo, sostiene que todo conocimiento es relativo y niega cualquier absoluto". (Ramírez, 2009, p.222). Esta plantea que la veracidad y validez del conocimiento puede variar de acuerdo a las condiciones espacio, temporales en las cuales se edificó, por tal razón, no hay una verdad absoluta y los puntos de vista son subjetivos encuadrados en la relatividad que experimenta la construcción del saber.

Ahora aparece en escena el constructivismo de Piaget, Ausubel, Vygotsky, quienes plantean que es el sujeto quien consolida de forma activa su propio conocimiento, en este sentido no se puede conocer una idea universal o específica, pues es solo la persona quien construye los esquemas en relación con sus presaberes y su entorno. Para Ramírez (2009), "El constructivismo concibe al sujeto-que-conoce y al objeto-por-conocer como entidades interdependientes y asume que la realidad es, en importante medida, hechura humana y por tanto el conocimiento solo puede ser construido bajo el control de algo que ya es conocido". (p.222)

Otro modelo que vale la pena mencionar es el evolucionista, autores como Lemarck y Mendel coinciden con la teoría de la evolución de las especies de charles Darwin pero en este caso aplicado a la teoría del conocimiento, donde se plantea que la condición de conocer esta dada por los cambios graduales que ha presentado la especie. "Así, el conocimiento resulta construido por el sujeto, o grupo de sujetos, para adaptarse a su ambiente con procesos realizados a diferentes niveles -biológico, psicológico o social- y su estructuración final deviene solo en el ajuste de partes preexistentes" (Ramírez, 2009, p.223). 


\section{REFERENCIAS BIBLIOGRÁFICAS}

Botero, A (2001). Diagnóstico filosófico de los paradigmas de enseñanza universitaria. Unipluri/versidad. Vol.1 No.2, 2001 -Versión Digital. Recuperado de: https://aprendeenlinea.udea. edu.co/revistas/index.php/unip/article/view/12393/11223

Chacón, A (2017). La génesis del conocimiento: de la sensación a la razón. Educere, vol. 21, núm. 69, 2017. Recuperado de: http://www.saber.ula.ve/bitstream/handle/123456789/44035/ articulo1.pdf?sequence $=1$ \&isAllowed $=\mathrm{y}$

Corominas, Johan (1987). Diccionario Etimológico de la lengua castellana. Credos, S.A. Madrid. Recuperado de: https://losapuntesdefilosofia.files.wordpress.com/2017/09/diccionarioetimolocc81 gico-abreviado-de-la-lengua-castellana-joan-corominas.pdf

Lazaro (2018). Principios educativos de la educación occidental: la Edad Media. Revista Brasileira de Educação, vol. 23, 2018. Recuperado de: https://www.redalyc.org/ jatsRepo/275/27554785017/html/index.html

Quintana, Jose (1982). CONCEPTO DE FILOSOFÍA EN EDUCACIÓN. Revista Española de Pedagogía, Año XI, N¹57. Recuperado de: https://revistadepedagogia.org/wp-content/ uploads/2018/04/4-Concepto-de-Filosof\%C3\%ADa-en-la-Educaci\%C3\%B3n.pdf

Ramírez, Augusto. (2009). La teoría del conocimiento en investigación científica: una visión actual. Anales de la Facultad de Medicina, 70(3), 217-224. Recuperado en 20 de marzo de 2020, de http://www.scielo.org.pe/scielo.php?script=sci_arttext\&pid=S1025-55832009000300011\&lng $=$ es\&tlng $=$ es.

Saeteros Pérez, Tamara (2013). Por mi alma subiré a Dios. El concepto de alma de san Agustín de Hipona. Civilizar. Ciencias Sociales y Humanas, 13(25),189-210.[fecha de Consulta 20 de Marzo de 2020]. ISSN: 1657-8953. Disponible en: https://www.redalyc.org/articulo. oa? id=1002/100230029011

Tamayo de Serrano, Clara (2007). El aporte cultural y educativo de la Baja Edad Media. Educación y Educadores, 10(2),197-213.[fecha de Consulta 20 de Marzo de 2020]. ISSN: 01231294. Disponible en: https://www.redalyc.org/articulo.oa?id=834/83410214 